\title{
Histone deacetylase enzyme silencing using shRNAs enhances radiosensitivity of SW579 thyroid cancer cells
}

\author{
YE WANG $^{1,2}$, TAO JIN ${ }^{2}$, XUEMING DAI ${ }^{2}$, DONGWANG YAN ${ }^{2}$ and ZHIHAI PENG ${ }^{1,2}$ \\ ${ }^{1}$ School of Medicine, Nanjing Medical University, Nanjing, Jiangsu 210029; ${ }^{2}$ Department of General Surgery, \\ Shanghai General Hospital, Shanghai 200080, P.R. China
}

Received April 3, 2015; Accepted April 15, 2016

DOI: $10.3892 / \mathrm{mmr} .2016 .5711$

\begin{abstract}
The aim of the present study was to screen the enzymes that are associated with the radiosensitivity of SW579 thyroid cancer cells, and investigate whether radiation, combined with specific RNA interference on the screened enzymes, enhances radiosensitivity of SW579 thyroid cancer cells. Quantitative polymerase chain reaction (qPCR) was used to analyze epigenetic enzyme expression changes before and after radiotherapy, and four enzymes, histone deacetylase 1 (HDAC1), HDAC2, HDAC4 and HDAC6 were screened. Western blot analysis was performed to analyze the change in HDAC1, HDAC2, HDAC4 and HDAC6 protein expression following radiotherapy. Short hairpin RNA (ShRNA)-HDAC1, shRNA-HDAC2, shRNA-HDAC4 and shRNA-HDAC6 plasmids were constructed and SW579 cells were transfected with corresponding shRNA-HDACs. Reverse transcription-qPCR was used to detect whether downregulation of HDAC mRNAs had been effective. In addition, shRNA and shRNA negative control (NC) pools were established and transfected into the SW579 cells. The samples were divided into four groups; control, trichostatin A, shRNA pool and shRNA NC pool, to analyze the effective enhancement of specific shRNA on radiosensitivity in thyroid cancer cells. The morphological changes were observed in the SW579 cells, and the number of tumor cells decreased markedly in the shRNA pool group compared with that of the other three groups. Therefore, it was concluded that HDACs present a potential target for increasing the sensitivity of thyroid cancer cells to radiotherapy, and shRNA-HDAC interference combined with radiotherapy promotes the radiosensitivity of tumors.
\end{abstract}

Correspondence to: Mr. Zhihai Peng, School of Medicine, Nanjing Medical University, 140 Hanzhong Road, Nanjing, Jiangsu 210029, P.R. China

E-mail: zhihaipeng0@163.com

Key words: thyroid cancer cells, radiotherapy, radiosensitivity, short hairpin RNA, histone deacetylase

\section{Introduction}

Thyroid cancer is the most common type of endocrine cancer worldwide and accounts for the majority of endocrine cancer-associated mortalities, although it represents only $1 \%$ of all malignant diseases $(1,2)$. Thyroid cancer-associated mortality results from tumor invasion with local and distant metastases (3). The following are at greater risk for developing thyroid cancer: Caucasian individuals; females; those with a low intake of iodine; and those that have previously been exposed to radiation (4). Furthermore, thyroid cancers are divided into three cancer categories; differentiated, medullary and anaplastic (5). The treatments for all types of anaplastic cancers include surgery, radioactive iodine treatment, thyroid hormone supplementation, external beam radiation therapy and chemotherapy (6).

Radiotherapy enables highly conformal treatment for thyroid cancer and has been demonstrated to improve overall survival $(7,8)$. In a previous study, external radiotherapy was administered to the thyroid bed and bilateral cervical lymphatics as part of initial therapeutic treatment (9). Furthermore, radiotherapy is often performed in combination with enzyme or gene inhibitors, or RNA interference (10). Radiotherapy was reported to be enhanced by cyclooxygenase- 2 enzyme inhibitors (10). Radiotherapy was performed with AZD2171 (a highly potent, orally active inhibitor of vascular endothelial growth factor receptor signaling) to treat lung cancer, and the result indicated that AZD2171 enhanced the antitumor effects of radiotherapy (11). It has also been used in combination with histone acetyltransferase and histone deacetylase (HDACs) inhibitors for the treatment of prostate, esophageal, and head and neck cancer (12).

Aberrant regulation of gene expression, attributed to alterations in HDAC recruitment and activity, has consistently been observed in solid and hematologic tumors (13). Therefore, HDACs can be considered as potential therapeutic targets for the treatment of human malignancies (14). HDACs are important in the maintenance and function of chromatin by regulating the acetylation state of histones (15). HDAC inhibitors are currently being evaluated as anticancer agents in clinical trials, which can induce growth arrest, differentiation and apoptosis of cancer cells in in vitro and in vivo tumor-bearing animal models (16-19). Trichostatin A (TSA) is a HDAC inhibitor and inhibits growth of small cell lung 
cancer cells (20). TSA may be administered therapeutically for the redifferentiation of thyroid cancers to promote radioiodide uptake (21). Double-stranded RNA-mediated interference (RNAi) has recently emerged as a notable tool in reverse genetic to silence gene expression in multiple types of organism, including plants, Caenorhabditis elegans and Drosophila (22). Furthermore, RNAi via the expression of shRNA molecules is considered to be a particularly promising tool in reverse genetics in mice, as it may enable inexpensive and rapid gene function analysis in vivo (23).

In the present study, reverse transcription-quantitative polymerase chain reaction (RT-qPCR) analysis was performed to screen the enzymes that are associated with the radiosensitivity of SW579 thyroid cancer cells. In addition, to evaluate the potential impact of the screened enzymes on SW579 cell radiosensitivity, shRNA-HDAC1, shRNA-HDAC2, shRNA-HDAC4, shRNA-HDAC6 plasmids were constructed and shRNA pools of the four shRNA plasmids were established. The cancer cells were transfected with shRNA pools and irradiated using X-rays. The morphology of cancer cells following radiotherapy was observed by fluorescence microscopy.

\section{Materials and methods}

Plasmid construction. Using HDAC1, HDAC2, HDAC4 and HDAC6 mRNA sequences that were obtained from GenBank (www.ncbi.nlm.nih.gov/genbank/) and shRNA design principles, as described in a previous study (22), the online design software (siRNA Selection Program; http://sirna.wi.mit.edu/), Ambion company, was used to establish the target sequences. Four shRNA plasmid sequences (shRNA-HDAC1, shRNA-HDAC2, shRNA-HDAC4 and shRNA-HDAC6) targeting different coding regions of human HDAC1, HDAC2, HDAC4 and HDAC6 mRNA were designed. In addition, four scramble sequence shRNA duplexes were synthesized to serve as negative controls (NCs) and were abbreviated as shRNA-HDAC1-NC, shRNA-HDAC2-NC, shRNA-HDAC4-NC and shRNA-HDAC6-NC. The sequences are presented in Table I. The shRNA pool, which contained shRNA-HDAC1, shRNA-HDAC2, shRNA-HDAC4 and shRNA-HDAC6 were constructed. The shRNA NC pool, which contained shRNA-HDAC1-NC, shRNA-HDAC2-NC, shRNA-HDAC4-NC and shRNA-HDAC6-NC was also established.

Cell culture and plasmid transfection. The SW579 human thyroid cancercells were obtained from the Shanghai Institutes for Biological Sciences, Chinese Academy of Sciences (Shanghai, China). They were cultured in $10 \mathrm{ml}$ Dulbecco's modified Eagle's medium (DMEM; Thermo Fisher Scientific, Inc., Waltham, MA, USA) supplemented with $10 \%$ fetal bovine serum (FBS; Thermo Fisher Scientific, Inc.). Prior to transfection $(12 \mathrm{~h}), \sim 2 \times 10^{5}$ cells $/ \mathrm{ml}$ cells were seeded in 6-well plates and $2 \mathrm{ml}$ culture medium (90\% DMEM and $10 \%$ FBS) was added to each well. Cells were grown overnight to 50-60\% confluence. All plasmids were transfected with $8 \mu \mathrm{l}$ metafectamine transfection reagent (Biontex Laboratories GmbH, Martinsried/Planegg, Germany) according to the manufacturer's instructions. shRNA expression plasmid
$(1 \mu \mathrm{g})$ and $8 \mu \mathrm{l}$ metafectamine were diluted in serum- and antibiotic-free OptiMEM medium (50 $\mu$ l; Thermo Fisher Scientific, Inc.). The plasmid and metafectamine solutions were combined and mixed by careful pipetting. Then the mixture was incubated at room temperature for $20 \mathrm{~min}$ and added to the wells containing the cells. The optiMEM was replaced with $2 \mathrm{ml}$ DMEM and 10\% FBS following incubation for $6 \mathrm{~h}$. Cells were cultured in a humidified atmosphere containing $5 \% \mathrm{CO}_{2}$ at $37^{\circ} \mathrm{C}$ for $72 \mathrm{~h}$. All transfection experiments were performed a minimum of three times. To analyze the downregulation of HDACs by shRNA, three groups were formed as follows: shRNA-HDAC1-1 (shRNA-HDAC2-1, shRNA-HDAC4-1 and shRNA-HDAC6-1); shRNA-HDAC1-2 (shRNA-HDAC2-2, shRNA-HDAC4-2 and shRNA-HDAC6-2); and shRNA-HDAC1-NC (shRNA-HDAC2-NC, shRNA-HDAC4-NC and shRNA-HDAC6-NC). To investigate the enhancement effect of shRNA pools on radiosensitivity of thyroid cancer cells, the samples were divided into four groups: Control (untransfected); TSA (Sigma-Aldrich, St. Louis, MO, USA); shRNA pool; and shRNA NC pool. The TSA group was differentiated from the control group by the addition of $200 \mu \mathrm{g} / 1$ HDAC inhibitor, TSA to the cell culture medium.

Irradiation of thyroid cancer cells. Log-phase tumor cells were seeded as single-cell suspensions into 6-well plates and were left to adhere overnight. The cells were irradiated with a single dose (8 Gy) of X-rays (FCS320; Gulmay Medical Ltd., Camberley, UK), and a standard colony-forming assay was performed, according to a previous study (24), to determine the respective surviving fractions. To estimate the sensitivity to radiotherapy, the cells were exposed to $3 \mu \mathrm{M}$ 5 -fluorouracil (Sigma-Aldrich) for $16 \mathrm{~h}$ before irradiation, as described by Spitzner et al (25). The relative mRNA levels were determined by comparing the values to mRNA levels prior to radiotherapy.

$R T-q P C R$. The expression changes of SW579 human thyroid cancer cell epigenetic enzymes before and after radiotherapy were analyzed by RT-PCR. The mRNA expression level of HDAC1, HDAC2, HDAC4 and HDAC6 in cancer cells after transfection with shRNA-HDAC1-1, shRNA-HDAC1-2, shRNA-HDAC1-NC, shRNA-HDAC2-1, shRNA-HDAC2-2, shRNA-HDAC2-NC, shRNA-HDAC4-1, shRNA-HDAC4-2, shRNA-HDAC4-NC, shRNA-HDAC6-1, shRNA-HDAC6-2 and shRNA-HDAC6-NC was also analyzed by RT-PCR.

Total RNA was isolated from the cultured cells using a TRIzol kit (Invitrogen; Thermo Fisher Scientific, Inc.). The RNA quality was assessed using the NanoDrop 1000 (Thermo Fisher Scientific, Inc.). RT-PCR was performed using QuantiTect Primer Assay (Qiagen GmbH, Hilden, Germany) and QuantiTect SYBR Green RT-PCR Kit (Qiagen GmbH) on a LightCycler 480 Instrument (Roche Diagnostics, Mannheim, Germany). The detection and quantification was performed as follows: Reverse transcription, $55^{\circ} \mathrm{C}$ for $30 \mathrm{~min}$; initial activation, $15 \mathrm{~min}$ at $95^{\circ} \mathrm{C} ; 40$ cycles of denaturation, $94^{\circ} \mathrm{C}$ for $15 \mathrm{sec}$; annealing, $30 \mathrm{sec}$ at $55^{\circ} \mathrm{C}$; and extension, $30 \mathrm{sec}$ at $72^{\circ} \mathrm{C}$. Fluorescence data was collected at the extension step. The relative expression of the target gene was determined using the $2^{-\Delta C t}$ method (26). 


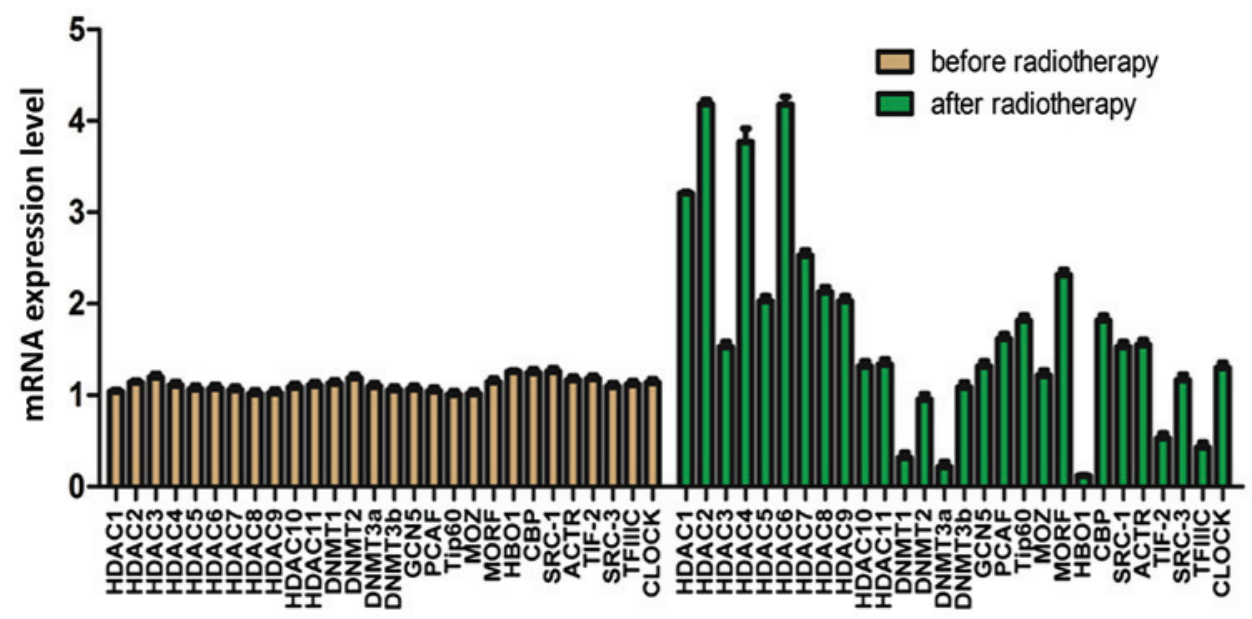

Figure 1. mRNA expression level of epigenetic enzymes in SW579 human thyroid cancer cells before and after radiotherapy by reverse transcription-quantitative polymerase chain reaction. HDAC, histone deacetylase.

Western blot analysis. Western blot analysis was performed on five groups of thyroid cancer cells as follows: i) Thyroid cancer cells before radiotherapy; ii) thyroid cancer cells after radiotherapy; iii) thyroid cancer cells transfected with shRNA-HDAC-1; iv) thyroid cancer cells transfected with shRNA-HDAC-2; and v) thyroid cancer cells transfected with shRNA-HDAC-NC. The cells were collected after transfection for $72 \mathrm{~h}$, and washed with cold phosphate-buffered saline (PBS; Sangon Biotech Co., Ltd., Shanghai, China) twice. The cells were lysed with $1 \mathrm{ml}$ lysis buffer (10\% SDS, $0.5 \%$ Bromophenol Blue, $50 \%$ glycerol, 5\% $\beta$-mercaptoethanol and $250 \mathrm{mmol} / 1$ Tris-HCl; pH 6.8; all obtained from Sangon Biotech Co., Ltd.) and phenylmethylsulfonyl fluoride (Sangon Biotech Co., Ltd.). The mixture was centrifuged at $198 \mathrm{x} \mathrm{g}$ for $5 \mathrm{~min}$ after boiling for $10 \mathrm{~min}$ and the supernatants were collected. The protein concentrations were determined according to the Pierce ${ }^{\mathrm{TM}}$ BCA Protein assay kit (Thermo Fisher Scientific, Inc.). The cell lysate was separated by $12 \%$ SDS-PAGE (Sangon Biotech Co., Ltd.) at $80 \mathrm{~V}$ for $30 \mathrm{~min}$, then $120 \mathrm{~V}$ for $100 \mathrm{~min}$ and transferred to nitrocellulose membranes (Mai Bio Co., Ltd., Shanghai, China) at $150 \mathrm{~mA}$ for $1 \mathrm{~h}$. After blocking non-specific binding sites with 5\% non-fat milk, the membrane was incubated with primary antibodies for $1.5 \mathrm{~h}$ at room temperature. The primary antibodies used in the experiment were mouse monoclonal anti-APE1 (1:3,000; Abcam, Cambridge, MA, USA; cat. no. ab194), rabbit polyclonal anti-HDAC1 (1:10,000; Abcam; cat. no. ab7028), rabbit monoclonal anti-HDAC2 (1:2,000; Abcam; cat. no. ab32117), rabbit polyclonal anti-HDAC4 (1:500; Abcam; cat.no. ab1437), rabbit polyclonal anti-HDAC6 (1:200; Abcam; cat. no. ab1440) and monoclonal anti- $\beta$-actin (1:5,000; Santa Cruz Biotechnology, Inc., Dallas, TX, USA; cat. no.sc-69879) antibodies. The membrane was washed with Tris-buffered saline and Tween-20 (Sangon Biotech Co., Ltd.) three times, for $10 \mathrm{~min}$ each time. The membrane was then incubated with horseradish peroxidase (HRP)-conjugated goat anti-rabbit IgG (1:5,000; Santa Cruz Biotechnology, Inc.; cat. no. sc-2054) and rabbit anti-mouse $\operatorname{IgG}(1: 5,000$; Santa Cruz Biotechnology, Inc.; cat. no. sc-358920) antibodies. Images of the membranes were captured (LAS-3000; Fujifilm, Tokyo, Japan), macroscopic observation was used to

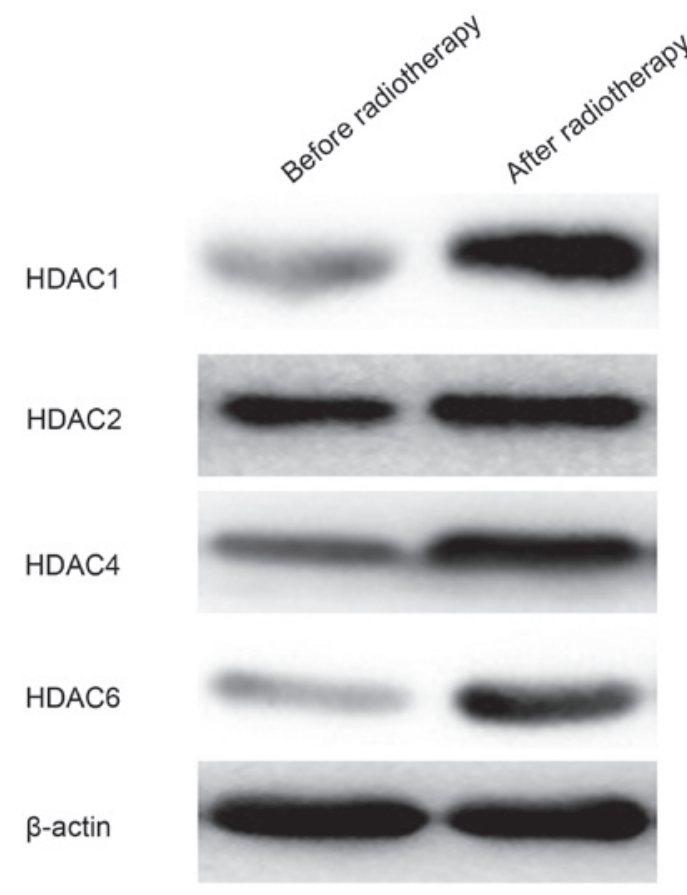

Figure 2. Western blot analysis of HDAC1, HDAC2, HDAC4 and HDAC6 protein expression in SW579 human thyroid cancer cells before and after radiotherapy. HDAC, histone deacetylase.

compare the western blotting results with an LAS-3000 and Multi Gauge (version 3.0; Fujifilm).

Morphology. Morphological analysis was performed on the four groups of thyroid cancer cells (thyroid cancer cells, cells cultured in medium with TSA, cells transfected with the shRNA pool and cells transfected with the shRNA NC pool). Cells were collected by centrifugation following two washes with PBS. Carboxyfluorescein (1:500; Beijing Fanbo Biochemicals Co., Ltd., Beijing, China) was used to mark the cells. Prior to observation, the cells were washed three times in PBS, agitated for $5 \mathrm{~min}$, dried and mounted with $30 \%$ glycerol. A fluorescence microscope (Q550CW; Leica Microsystems $\mathrm{GmbH}$, Wetzlar, Germany; magnification, x10) with Cooke 
Table I. The mRNA sequence of different shRNA-HDAC short fragment.

\begin{tabular}{|c|c|}
\hline Index & Primer sequence \\
\hline shRNA-HDAC1-1 & $\begin{array}{l}\text { F: 5'-CACCGCTCCATCCGTCCAGATAACACGAATGTTATCTGGACGGATGGAGC-3' } \\
\text { R: 5'-AAAAGCTCCATCCGTCCAGATAACATTCGTGTTATCTGGACGGATGGAGC-3' }\end{array}$ \\
\hline shRNA-HDAC1-2 & $\begin{array}{l}\text { F: 5'-CACCGCAAGCAGATGCAGAGATTCACGAATGAATCTCTGCATCTGCTTGC-3' } \\
\text { R: 5'-AAAAGCAAGCAGATGCAGAGATTCATTCGTGAATCTCTGCATCTGCTTGC-3' }\end{array}$ \\
\hline shRNA-HDAC1-NC & $\begin{array}{l}\text { F: 5'-CACCGCAATTTTTTTTTTTGATTCACGAATGAATCAAAAAAAAAAAAATGC-3' } \\
\text { R: 5'-AAAAGCAATTTTTTTTTTTGATTCATTCGTGAATCAAAAAAAAAAAAATGC-3 }\end{array}$ \\
\hline shRNA-HDAC2-1 & $\begin{array}{l}\text { F: 5'-CACCGCAGATGCAGAGATTTAATGTCGAAACATTAAATCTCTGCATCTGC-3' } \\
\text { R: 5'-AAAAGCAGATGCAGAGATTTAATGTTTCGACATTAAATCTCTGCATCTGC-3' }\end{array}$ \\
\hline shRNA-HDAC2-2 & $\begin{array}{l}\text { F: 5'-CACCGGCTGGAGGATTACATCATGCCGAAGCATGATGTAATCCTCCAGCC-3' } \\
\text { R: 5'-AAAAGGCTGGAGGATTACATCATGCTTCGGCATGATGTAATCCTCCAGCC-3' }\end{array}$ \\
\hline shRNA-HDAC2-NC & $\begin{array}{l}\text { F: 5'-CACCGCAATTTTTTTTTTTGATTCACGAATGAATCAAAAAAAAAAAAATGC-3' } \\
\text { R: 5'-AAAAGCAATTTTTTTTTTTGATTCATTCGTGAATCAAAAAAAAAAAAATGC-3 }\end{array}$ \\
\hline shRNA-HDAC4-1 & $\begin{array}{l}\text { F: 5'-CACCGGAGCTCGTGGTACTCAAACACGAATGTTTGAGTACCACGAGCTCC-3' } \\
\text { R: 5'-AAAAGGAGCTCGTGGTACTCAAACATTCGTGTTTGAGTACCACGAGCTCC-3' }\end{array}$ \\
\hline shRNA-HDAC4-2 & $\begin{array}{l}\text { F: 5'-CACCGGAACACATCAAGCACCAACACGAATGTTGGTGCTTGATGTGTTCC-3' } \\
\text { R: 5'-AAAAGGAACACATCAAGCACCAACATTCGTGTTGGTGCTTGATGTGTTCC-3' }\end{array}$ \\
\hline shRNA-HDAC4-NC & $\begin{array}{l}\text { F: 5'-CACCGCAATTTTTTTTTTTGATTCACGAATGAATCAAAAAAAAAAAAATGC-3' } \\
\text { R: 5'-AAAAGCAATTTTTTTTTTTGATTCATTCGTGAATCAAAAAAAAAAAAATGC-3 }\end{array}$ \\
\hline shRNA-HDAC6-1 & $\begin{array}{l}\text { F: 5'-CACCGCAATGGAAGAAGACCTAATCCGAAGATTAGGTCTTCTTCCATTGC-3' } \\
\text { R: 5'-AAAAGCAATGGAAGAAGACCTAATCTTCGGATTAGGTCTTCTTCCATTGC-3' }\end{array}$ \\
\hline shRNA-HDAC6-2 & $\begin{array}{l}\text { F: 5'-CACCGGAAGAGCTGATGTTGGTTCACGAATGAACCAACATCAGCTCTTCC-3' } \\
\text { R: 5'-AAAAGGAAGAGCTGATGTTGGTTCATTCGTGAACCAACATCAGCTCTTCC-3' }\end{array}$ \\
\hline siRNA-HDAC6-NC & $\begin{array}{l}\text { F: 5'-CACCGCAATTTTTTTTTTTGATTCACGAATGAATCAAAAAAAAAAAAATGC-3' } \\
\text { R: 5'-AAAAGCAATTTTTTTTTTTGATTCATTCGTGAATCAAAAAAAAAAAAATGC-3 }\end{array}$ \\
\hline$\beta$-actin & $\begin{array}{l}\text { F: 5'-GTGGACATCCGCAAAGAC-3' } \\
\text { R: 5'-AAAGGGTGTAACGCAACTA-3' }\end{array}$ \\
\hline
\end{tabular}

shRNA, short hairpin RNA; HDAC, histone deacetylase; NC, negative control; F, forward; R, reverse.

software version 3.04 (cooke-viewer.software.informer.com) was used to perform apoptosis analysis from total cell and living cell numbers counted.

Statistical analysis. All data were expressed as the mean \pm standard deviation from a minimum of three separate experiments. The differences between groups were analyzed using Student's t-test in the SPSS 10.0 software (SPSS, Inc., Chicago, IL, USA). $\mathrm{P}<0.05$ was considered to indicate a statistically significant difference.

\section{Results}

Epigenetic enzyme expression level changes after radiotherapy. RT-PCR was used to analyze the epigenetic enzyme expression changes in SW579 human thyroid cancer cells following radiotherapy. The results are presented in Fig. 1. For the majority of the enzymes, the mRNA expression level after radiotherapy was higher compared with before irradiation. The increase was particularly notable in HDAC1, HDAC2, HDAC4 and HDAC6.
To further confirm the change in HDAC1, HDAC2, HDAC4 and HDAC6 protein expression, western blot analysis was performed before and after radiotherapy (Fig. 2). The protein expression level of HDAC1, HDAC2 and HDAC6 in cells after irradiation was markedly higher compared with before irradiation. The protein expression intensity of HDAC4 was also greater in cells after irradiation. These findings demonstrated that radiotherapy upregulated the expression level of HDAC1, HDAC2, HDAC4 and HDAC6 proteins.

Inhibition of HDAC expression after transfection with shRNA-HDAC. ShRNA-HDAC1-1, shRNA-HDAC1-2, shRNA-HDAC1-NC, shRNA-HDAC2-1, shRNA-HDAC2-2, shRNA-HDAC2-NC, shRNA-HDAC4-1, shRNA-HDAC4-2, shRNA-HDAC4-NC, shRNA-HDAC6-1, shRNA-HDAC6-2 and shRNA-HDAC6-NC plasmids were constructed, and transfected into cultured SW579 thyroid cancer cells. To analyze the inhibition effect of shRNA-HDAC plasmid on HDAC expression in the SW579 cells, RT-PCR and western blot analysis were conducted (Fig. 3). As shown, the mRNA expression level of HDAC1, HDAC2, HDAC4 and HDAC6 in 
A

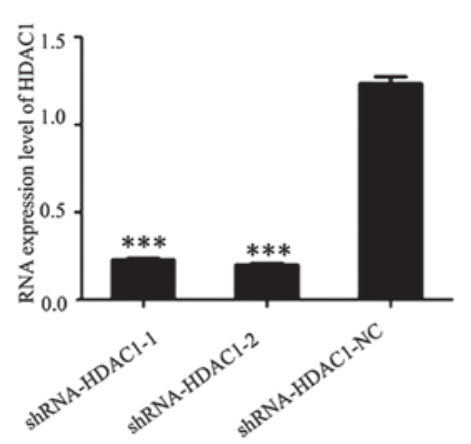

C

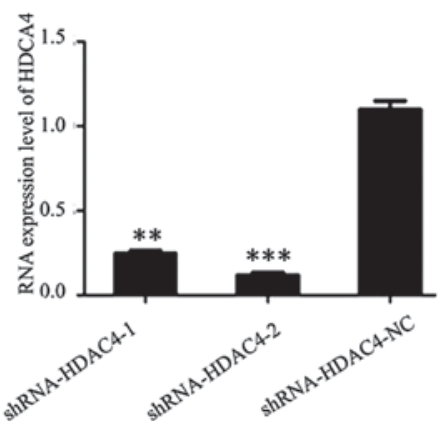

B

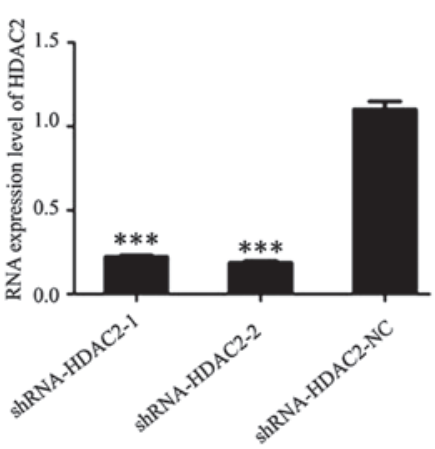

D

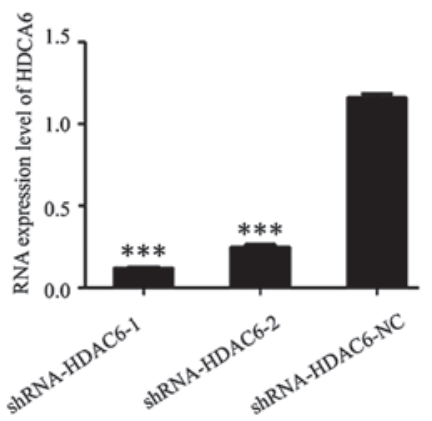

Figure 3. mRNA expression level of (A) HDAC1; (B) HDAC2; (C) HDAC4; and (D) HDAC6 following transfection with specific shRNAs as analyzed by reverse transcription-quantitative polymerase chain reaction. ${ }^{* *} \mathrm{P}<0.01,{ }^{* * *} \mathrm{P}<0.001$ vs. the shRNA-HDAC6-NC group. shRNA, short hairpin RNA; HDAC, histone deacetylase; NC, negative control.

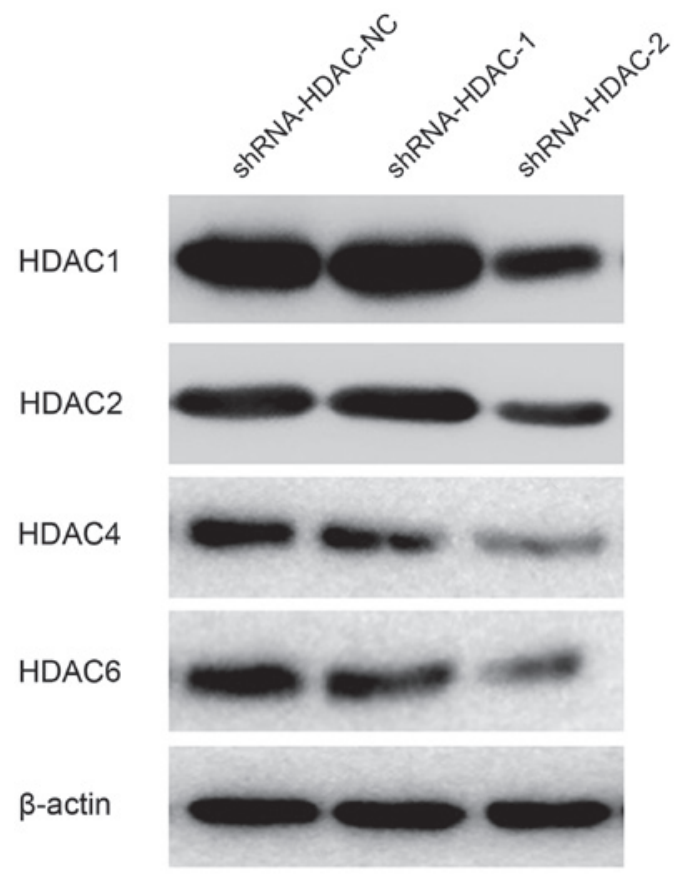

Figure 4. HDAC1, HDAC2, HDAC4 and HDAC6 protein expression following transfection with specific shRNAs, as analyzed by reverse transcription-quantitative polymerase chain reaction. shRNA, short hairpin RNA; HDAC, histone deacetylase.

thyroid cancer cells was markedly decreased after transfection with shRNA-HDAC1, shRNA-HDAC2, shRNA-HDAC4 and shRNA-HDAC6 plasmids, when compared with those that were transfected with shRNA-HDAC1-NC, shRNA-HDAC2-NC, shRNA-HDAC4-NC and shRNA-HDAC6-NC (Fig. 4). The protein expression in the positive interference group (shRNA-HDAC-1 and shRNA-HDAC-2) was significantly weaker when compared with the negative interference group (shRNA-HDAC-NC); the $\beta$-actin expression in the three groups was similar.

Radiotherapy following transfection with the shRNA pool. To investigate whether an shRNA pool plasmid strengthens the sensitivity of SW579 cells to radiotherapy, comparisons 


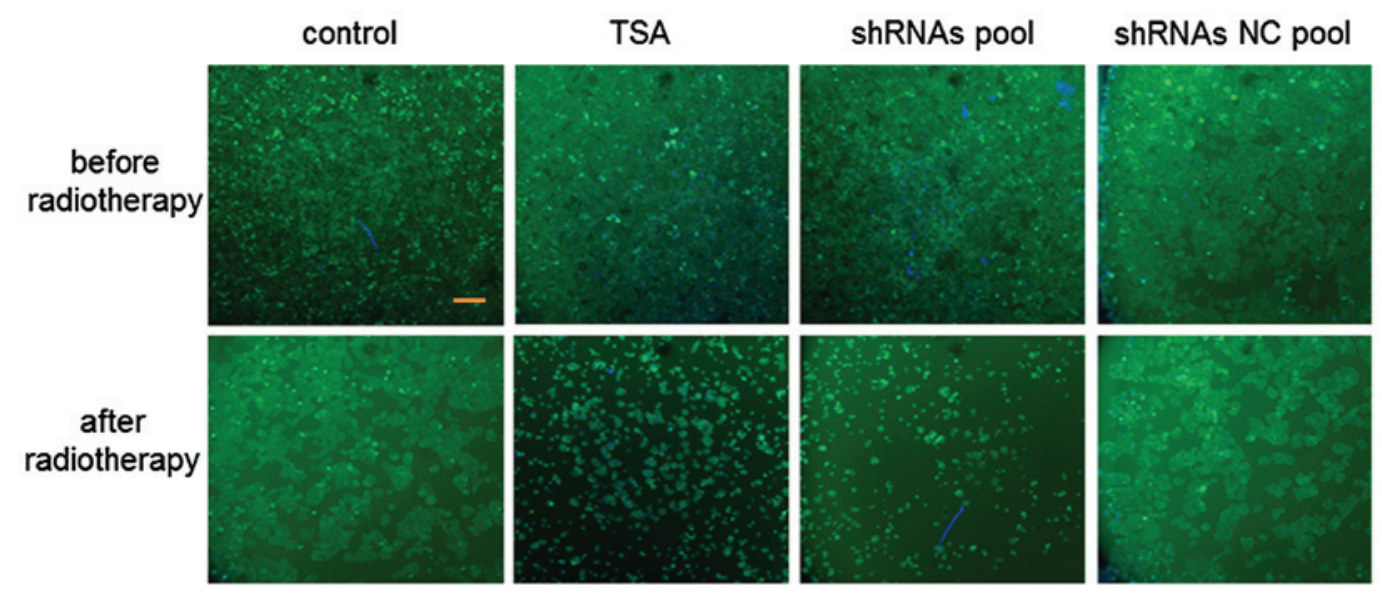

Figure 5. Morphology of sw579 human thyroid cancer cells, before and after radiotherapy, by fluorescence microscopy. Scale bar, $100 \mu \mathrm{m}$. TSA, trichostatin A; shRNA, short hairpin RNA; NC, negative control.

between the thyroid cancer cell morphology in the control, TSA, shRNA pool and shRNAs NC pool groups was performed (Fig. 5). The cell number in the control and shRNA NC pool groups was notably higher than that of the TSA and shRNA pool groups, which demonstrated that apoptosis was promoted in the TSA and shRNA pool groups. Furthermore, the cell number in the shRNA pool group was markedly lower than that of the TSA group, indicating that the shRNA pool strengthened the sensitivity of SW579 thyroid cancer cells to a greater extent than the HDAC inhibitor, TSA.

\section{Discussion}

Thyroid cancer is associated with a high mortality rate. Throughout the past two decades, an increasing incidence of thyroid cancer has been reported worldwide (27). In the present study, RT-PCR was used to analyze the epigenetic enzyme expression level changes in SW579 thyroid cancer cells to investigate treatment of thyroid cancer at the molecular level. The result demonstrated that the expression level of HDAC1, HDAC2, HDAC4 and HDAC6 was markedly increased following radiotherapy. It was hypothesized that HDAC1, HDAC2, HDAC4 and HDAC6 expression may be associated with the radiosensitivity of thyroid cancer cells and may be key in the regulation of cellular radiosensitivity. Western blot analysis was performed to further analyze the protein expression level of the four enzymes, and the result indicated that the expression intensity of HDAC1, HDAC2, HDAC4 and HDAC6 following radiotherapy was markedly stronger than before radiotherapy. HDACs exert antagonistic actions on histones, and regulate chromatin structure and function by catalyzing removal of the acetyl modification from the lysine residues of histones $(28,29)$. When HDACs act upon nucleosomal histones, it results in the tight coiling of chromatin and silencing of the expression of various genes, which include those implicated in the regulation of cell survival, proliferation, differentiation, and apoptosis (30). HDAC1, HDAC2, HDAC3 and HDAC8 are key in regulating cell proliferation and are expressed almost exclusively in the nucleus of the majority of cell types (31). Overexpression of HDACs has been reported in certain types of cancer tissue, such as stomach, colorectal, breast, and lung (32-35). Therefore, the present study proposed that HDAC1, HDAC2, HDAC4 and HDAC6 were associated with the radiosensitivity of SW579 thyroid cancer cells.

HDAC activity has been reported to be aberrant in numerous types of cancer, therefore, HDAC inhibitors have been adopted as potential anticancer therapies (36). Various HDAC inhibitors have been developed and TSA is one example. In a previous study, TSA induced apoptosis and cell-cycle arrest in anaplastic thyroid cancer cells (37). The use of TSA to treat thyroid cancer has been hypothesized to increase the efficacy of radioactive iodine therapy (38). Furthermore, TSA, when combined with radiotherapy, may induce cell death in medulloblastoma (39). In recent years, RNAi has developed rapidly and become an effective and useful technique in molecular biology and oncology; particularly regarding human gene function, signal transduction research and gene therapy (40). At present, RNAi is employed to downregulate the expression of specific genes, which have been targeted in the gene therapy of neurodegenerative diseases, Huntington's disease and the HIV infection (41-43). In the present study, shRNA-HDAC1, shRNA-HDAC1-NC, shRNA-HDAC2, shRNA-HDAC2-NC, shRNA-HDAC4, shRNA-HDAC4-NC, shRNA-HDAC6 and shRNA-HDAC6-NC plasmids were successfully constructed. Then, shRNA and shRNA NC pools of these plasmids were established, and apoptosis analysis was performed on four groups (control, TSA, shRNA pool and shRNA NC pool). In the shRNA pool and shRNA NC pool groups, SW579 thyroid cancer cells were transfected with the shRNA pool and shRNA NC pools, respectively. The result demonstrated that the number of living cells in the TSA and shRNA pool groups was markedly smaller than in the control and shRNA NC pool groups. Furthermore, the number of cells observed in the shRNA pool group was markedly lower when compared with the TSA group. This finding demonstrated that TSA and specific shRNA enhanced the sensitivity of SW579 thyroid cancer cells to radiotherapy; furthermore, the effect of radiotherapy enhanced by specific shRNA was greater than by TSA. In a previous study, U251 glioma cells were transfected with shRNA-PGK1 and the result indicated that tumor growth of the U251 xenografts was significantly inhibited, which indicated the downregulation of PGK1 (resulting from 
transfection with shRNA) had sensitized the U251 xenografts to radiotherapy (44). Wang et al (45) reported that the silencing of signal transducer and activator of transcription 3 expression using RNAi enhanced the efficacy of radiotherapy for laryngeal carcinoma in vivo. In human glioblastoma, the transfection of lentivirus-mediated shRNA to glioblastoma cells was reported to inhibit NADPH oxidase 4, which assisted with overcoming the radioresistance of cells and improved its therapeutic efficacy for glioblastoma (46). In colorectal cancer, the silencing of Wnt transcription factor, transcription factor 4 by specific shRNA sensitized colorectal cancer cells to (chemo-) radiotherapy (47). Therefore, the present study concluded that silencing HDAC1, HDAC2, HDAC4 and HDAC6 using an shRNA pool enhanced the sensitivity of SW579 thyroid cancer cells to radiotherapy and the enhanced effect was more marked than that of the HDAC inhibitor, TSA.

In conclusion, the enzymes, HDAC1, HDAC2, HDAC4 and HDAC6 in SW579 thyroid cancer cells were sensitive to radiotherapy, and their expression level was markedly increased following radiotherapy. The transfection of an shRNA pool, which was established using shRNA-HDAC1, shRNA-HDAC2, shRNA-HDAC4 and shRNA-HDAC6 in the SW579 thyroid cancer cells, enhanced their radiosensitivity. Thus, shRNA-targeted HDAC1, HDAC2, HDAC4 and HDAC6 may present as a potential strategy for gene therapy of thyroid cancer.

\section{References}

1. Mitsiades CS, Poulaki V, McMullan C, Negri J, Fanourakis G, Goudopoulou A, Richon VM, Marks PA and Mitsiades N: Novel histone deacetylase inhibitors in the treatment of thyroid cancer. Clin Cancer Res 11: 3958-3965, 2005.

2. Abu-Amero KK, Alzahrani AS, Zou M and Shi Y: High frequency of somatic mitochondrial DNA mutations in human thyroid carcinomas and complex I respiratory defect in thyroid cancer cell lines. Oncogene 24: 1455-1460, 2005.

3. Roomi MW, Bhanap B, Niedzwiecki A and Rath M: Inhibitory effects of a novel nutrient mixture on MMP secretion and invasion on human thyroid cancer cell line SW 579. JANA 12: 26-34, 2009

4. McTiernan A, Weiss NS and Daling JR: Incidence of thyroid cancer in women in relation to known or suspected risk factors for breast cancer. Cancer Res 47: 292-295, 1987.

5. Pasieka JL: Anaplastic thyroid cancer. Curr Opin Oncol 15: 78-83, 2003

6. O'Doherty MJ and Coakley AJ: Drug therapy alternatives in the treatment of thyroid cancer. Drugs 55: 801-812, 1998.

7. Rosenbluth BD, Serrano V, Happersett L, Shaha AR, Tuttle RM, Narayana A, Wolden SL, Rosenzweig KE, Chong LM and Lee NY: Intensity-modulated radiation therapy for the treatment of nonanaplastic thyroid cancer. Int J Radiat Oncol Biol Phys 63: 1419-1426, 2005

8. Wang Y, Tsang R, Asa S, Dickson B, Arenovich T and Brierley J: Clinical outcome of anaplastic thyroid carcinoma treated with radiotherapy of once-and twice-daily fractionation regimens. Cancer 107: 1786-1792, 2006.

9. Chow SM, Law SC, Mendenhall WM, Au SK, Chan PT, Leung TW, Tong CC, Wong IS and Lau WH: Papillary thyroid carcinoma: Prognostic factors and the role of radioiodine and external radiotherapy. Int J Radiat Oncol Biol Phys 52: 784-795, 2002.

10. Choy $\mathrm{H}$ and Milas L: Enhancing radiotherapy with cyclooxygenase-2 enzyme inhibitors: A rational advance? J Natl Cancer Inst 95: 1440-1452, 2003.

11. Williams KJ, Telfer BA, Shannon AM, Babur M, Stratford IJ and Wedge SR: Combining radiotherapy with AZD2171, a potent inhibitor of vascular endothelial growth factor signaling: pathophysiologic effects and therapeutic benefit. Mol Cancer Ther 6: 599-606, 2007.
12. Coucke P: A phase I trial on LBH 589 (panobinostat), a histone deacetylase inhibitor (HDAC) in combination with external radiotherapy for the treatment of prostate cancer, esophageal cancer and head and neck cancer. BJMO 3: 117-123, 2009.

13. Minucci S and Pelicci PG: Histone deacetylase inhibitors and the promise of epigenetic (and more) treatments for cancer. Nat Rev Cancer 6: 38-51, 2006.

14. Borbone E, Berlingieri MT, De Bellis F, Nebbioso A, Chiappetta G, Mai A, Altucci L and Fusco A: Histone deacetylase inhibitors induce thyroid cancer-specific apoptosis through proteasome-dependent inhibition of TRAIL degradation. Oncogene 29: 105-116, 2010.

15. Thurn KT, Thomas S, Moore A and Munster PN: Rational therapeutic combinations with histone deacetylase inhibitors for the treatment of cancer. Future Oncol 7: 263-283, 2011.

16. Gallinari P, Di Marco S, Jones P, Pallaoro M and Steinkühler C: HDACs, histone deacetylation and gene transcription: From molecular biology to cancer therapeutics. Cell Res 17: 195-211, 2007.

17. Giaginis C, Alexandrou P, Delladetsima I, Giannopoulou I, Patsouris E and Theocharis S: Clinical significance of histone deacetylase (HDAC)-1, HDAC-2, HDAC-4 and HDAC-6 expression in human malignant and benign thyroid lesions. Tumour Biol 35: 61-71, 2014.

18. Shen L, Orillion A and Pili R: Histone deacetylase inhibitors as immunomodulators in cancer therapeutics. Epigenomics 8: 415-428, 2016

19. Wang L, Bao X, Yang J, Li H, Zhou Q, Jiang X, Li M, Liu X, Yuan X, Sun Y, et al: Novel cinnamohydroxamic acid derivatives as HDAC inhibitors with anticancer activity in vitro and in vivo. Chem Biol Interact 249: 64-70, 2016.

20. Platta CS, Greenblatt DY, Kunnimalaiyaan M and Chen H: The HDAC inhibitor trichostatin A inhibits growth of small cell lung cancer cells. J Surg Res 142: 219-226, 2007.

21. Clarke C, Burbridge E and Smyth P: Can deacetylation promote radioiodide uptake in thyroid cancer? Endocrine Abstracts 9: 154, 2005.

22. Sui G, Soohoo C, Affar el B, Gay F, Shi Y, Forrester WC and Shi Y: A DNA vector-based RNAi technology to suppress gene expression in mammalian cells. Proc Natl Acad Sci USA 99: 5515-5520, 2002.

23. Seibler J, Küter-Luks B, Kern H, Streu S, Plum L, Mauer J, Kühn R, Brüning JC and Schwenk F: Single copy shRNA configuration for ubiquitous gene knockdown in mice. Nucleic Acids Res 33: e67, 2005.

24. Wu G, Ma Z, Qian J and Liu B: PP4R1 accelerates cell growth and proliferation in HepG2 hepatocellular carcinoma. Onco Targets Ther 8: 2067-2074, 2015.

25. Spitzner M, Emons G, Kramer F, Gaedcke J, Rave-Fränk M, Scharf JG, Burfeind P, Becker H, Beissbarth T, Ghadimi BM, et al: A gene expression signature for chemoradiosensitivity of colorectal cancer cells. Int J Radiat Oncol Biol Phys 78: 1184-1192, 2010

26. Livak KJ and Schmittgen TD: Analysis of relative gene expression data using real-time quantitative PCR and the 2(-Delta Delta C(T)) Method. Methods 25: 402-408, 2001.

27. Elashoff M, Matveyenko AV, Gier B, Elashoff R and Butler PC: Pancreatitis, pancreatic and thyroid cancer with glucagon-like peptide-1-based therapies. Gastroenterology 141: 150-156, 2011.

28. Kelly WK, O'Connor OA, Krug LM, Chiao JH, Heaney M, Curley T, MacGregore-Cortelli B, Tong W, Secrist JP, Schwartz L, et al: Phase I study of an oral histone deacetylase inhibitor, suberoylanilide hydroxamic acid, in patients with advanced cancer. J Clin Oncol 23: 3923-3931, 2005.

29. Luong QT, O'Kelly J, Braunstein GD, Hershman JM and Koeffler HP: Antitumor activity of suberoylanilide hydroxamic acid against thyroid cancer cell lines in vitro and in vivo. Clin Cancer Res 12: 5570-5577, 2006.

30. Jones PA and Baylin SB: The fundamental role of epigenetic events in cancer. Nat Rev Genet 3: 415-428, 2002.

31. Khochbin S, Verdel A, Lemercier C and Seigneurin-Berny D: Functional significance of histone deacetylase diversity. Curr Opin Genet Dev 11: 162-166, 2001.

32. Kim JH, Choi YK, Kwon HJ, Yang HK, Choi JH and Kim DY: Downregulation of gelsolin and retinoic acid receptor beta expression in gastric cancer tissues through histone deacetylase 1. J Gastroenterol Hepatol 19: 218-224, 2004.

33. Giannini R and Cavallini A: Expression analysis of a subset of coregulators and three nuclear receptors in human colorectal carcinoma. Anticancer Res 25: 4287-4292, 2005. 
34. Krusche CA, Wülfing $P$, Kersting C, Vloet A, Böcker W, Kiesel L, Beier HM and Alfer J: Histone deacetylase-1 and-3 protein expression in human breast cancer: A tissue microarray analysis. Breast Cancer Res Treat 90: 15-23, 2005.

35. Sasaki H, Moriyama S, Nakashima Y, Kobayashi Y, Kiriyama M, Fukai I, Yamakawa Y and Fujii Y: Histone deacetylase 1 mRNA expression in lung cancer. Lung Cancer 46: 171-178, 2004.

36. Woyach JA, Kloos RT, Ringel MD, Arbogast D, Collamore M, Zwiebel JA, Grever M, Villalona-Calero M and Shah MH: Lack of therapeutic effect of the histone deacetylase inhibitor vorinostat in patients with metastatic radioiodine-refractory thyroid carcinoma. J Clin Endocrinol Metab 94: 164-170, 2009.

37. Furuya F, Shimura H, Suzuki H, Taki K, Ohta K, Haraguchi K, Onaya T, Endo T and Kobayashi T: Histone deacetylase inhibitors restore radioiodide uptake and retention in poorly differentiated and anaplastic thyroid cancer cells by expression of the sodium/iodide symporter thyroperoxidase and thyroglobulin. Endocrinology 145: 2865-2875, 2004.

38. Zarnegar R, Brunaud L, Kanauchi H, Wong M, Fung M, Ginzinger D, Duh QY and Clark OH: Increasing the effectiveness of radioactive iodine therapy in the treatment of thyroid cancer using Trichostatin A, a histone deacetylase inhibitor. Surgery 132: 984-990, 2002.

39. Kumar KS, Sonnemann J, Hong le TT, Buurman C, Adler F, Maass M, Völker U and Beck JF: Histone deacetylase inhibitors, but not vincristine, cooperate with radiotherapy to induce cell death in medulloblastoma. Anticancer Res 27: 465-470, 2007.
40. Wang J,MiP,Lin G,Wang YX,Liu G and Chen X: Imaging-guided delivery of RNAi for anticancer treatment. Adv Drug Deliv Rev: Jan 22, 2016 (Epub ahead of print).

41. Bennasser Y, Yeung ML and Jeang KT: RNAi therapy for HIV infection principles and practicalities. BioDrugs 21: 17-22, 2007.

42. Harper SQ: Progress and challenges in RNA interference therapy for Huntington disease. Arch Neurol 66: 933-938, 2009.

43. Boudreau RL and Davidson BL: RNAi therapy for neurodegenerative diseases. Curr Top Dev Biol 75: 73-92, 2006.

44. Cheng YJ, Ding H, Du HQ, Yan H, Zhao JB, Zhang WB, Zou YJ, Liu HY and Xiao H: Downregulation of phosphoglycerate kinase 1 by shRNA sensitizes U251 xenografts to radiotherapy. Oncol Rep 32: 1513-1520, 2014.

45. Wang HR, Li XM and Lu XY: Silencing of signal transducer and activator of transcription 3 gene expression using RNAi enhances the efficacy of radiotherapy for laryngeal carcinoma in vivo. Zhonghua Er Bi Yan Hou Tou Jing Wai Ke Za Zhi 44: 591-596, 2009 (In Chinese).

46. Li Y, Han N, Yin T, Huang L, Liu S, Liu D, Xie C and Zhang M: Lentivirus-mediated Nox4 shRNA invasion and angiogenesis and enhances radiosensitivity in human glioblastoma. Oxid Med Cell Longev 2014: 581732, 2014.

47. Kendziorra E, Ahlborn K, Spitzner M, Rave-Fränk M, Emons G, Gaedcke J, Kramer F, Wolff HA, Becker H, Beissbarth T, et al: Silencing of the Wnt transcription factor TCF4 sensitizes colorectal cancer cells to (chemo-) radiotherapy. Carcinogenesis 32: 1824-1831, 2011. 